\title{
Advances in bile acids-mediated liver injury and liver regeneration
}

\author{
Xiaowen Tang ${ }^{1}$, Lili Ding ${ }^{1}$, Qiaoling Yang ${ }^{1}$, Xiaoyuan Niu ${ }^{1}$, Li Yang ${ }^{1,2, ~}{ }^{*}$, Zhengtao Wang ${ }^{1}$ \\ ${ }^{1}$ The MOE Key Laboratory for Standardization of Chinese Medicines and the SATCM Key Laboratory for New Resources and Quality \\ Evaluation of Chinese Medicines, Institute of Chinese Materia Medica, Shanghai University of Traditional Chinese Medicine, Shanghai, \\ China \\ ${ }^{2}$ Center for Chinese Medical Therapy and Systems Biology, Shanghai University of Traditional Chinese Medicine, Shanghai, China
}

\section{Email address:}

shs912vv@126.com (X. Tang), nai18219@126.com (L. Ding), cherish0814@126.com (Q. Yang), magicnxy@126.com (X. Niu) yangli7951@hotmail.com (L. Yang,),wangzht@hotmail.com (Z. Wang)

\section{To cite this article:}

Xiaowen Tang, Lili Ding, Qiaoling Yang, Xiaoyuan Niu, Li Yang, Zhengtao Wang. Advances in Bile Acids-Mediated Liver Injury and Liver Regeneration. Advances in Biochemistry. Vol. 2, No. 6, 2014, pp. 85-89. doi: 10.11648/j.ab.20140206.11

\begin{abstract}
Bile acids are endogenous molecules that originate from the liver and transport via bile to the intestines. They normally regulate cholesterol homeostasis, stimulate lipid solubilization and mediate metabolic signaling. Early studies implicated that disorders of bile acids compositions and concentrations can cause liver injury. Several hydrophobic bile acids are toxic and ample increases of them in liver may induce cell inflammation, apoptosis and necrosis. While the hydrophilic bile acid, such as ursodeoxycholic acid, has a therapeutic effect on cholestatic liver diseases. Further more, recent researches demonstrate that bile acids have also been implicated in stimulation of liver regeneration. The antagonistic regulation of liver injury and liver regeneration by bile acids may correlate with its composition and concentration. This review will focus on both how different bile acids and different bile acid concentrations play a critical role in liver injury and regeneration.
\end{abstract}

Keywords: Bile Acids, Liver Injury, Liver Regeneration, UDCA

\section{Introduction}

Bile acids, which originate from cholesterol, are amphipathic molecules that not only facilitate the uptake of lipids but also interact with the aqueous environment. The biological functions of BAs are studied intensively in the past decades. As endogenous molecules, BAs regulate energy homeostases, activate nuclear receptors and control cell proliferations and inflammatory processes in the liver ${ }^{[1-3]}$. It has been well studied that several secondary BAs such as lithocholic acid (LCA) and deoxycholic acid (DCA) are cytotoxic and have a potential to lead to hepatocellular injury, which may cause severe inflammation, apoptosis and necrosis later and develop to malignant tumor in the end ${ }^{[3,4]}$.

However, recent evidences suggest that bile acids also stimulate liver regeneration ${ }^{[5-7]}$. Liver regeneration has been elaborated since the generation of a partial hepatectomy $(\mathrm{PH})$ model on rats in 1931. Normally, it starts with quiescent hepatocytes undergoing one or two rounds of replication to restore the liver mass and involves a complex interaction among cytokines, growth factors and metabolics ${ }^{[8]}$. A recent literature is replete with data and displays that bile acids may promote liver regeneration through nuclear receptor-dependent signaling ${ }^{[9]}$ so that more and more investigators realize that the abnormal compositions and concentrations of hepatic BAs not only trigger the beginning of liver damage but also may be the start of self-healing.

Ursofalk is first patent medicine that stems from bile acid analogues. Its main ingredient is ursodeoxycholic acid (UDCA), which is now routinely used for treatment of primary biliary cirrhosis (PBC), primary sclerosing cholangitis (PSC) and intrahepatic cholestasis in pregnancy with little side effects ${ }^{[10]}$. Two more bile acid analogues (6-ECDCA and TUDCA) were proved effective in cholestasic liver disease during the past decades ${ }^{[11,12]}$. With the deepening of the researches, many investigators proposed that BAs may possess hormone-like effects in regulating liver function and have a unique potential to develop new drugs due to its multiple effects. 


\section{Enterohepatic Circulation of Bile Acids}

Bile acids convert from cholesterol with the help of 17 key enzymes ${ }^{[13]}$. In human beings, chenodeoxycholate acid (CDCA) and cholate acid (CA) are the primary bile acids synthesized in the liver. Later, they are conjugated to taurine or glycine to increase their water solubility ${ }^{[14]}$. Generally, the ratio of glycine conjugates to taurine conjugates in humans is nearly 1: 3 due to the relative abundance of the two amino acids. While in rodents, there are rare glycine bile salts ${ }^{[15]}$. The conjugated BAs mediate bile flow and form mixed micelles with phospholipids to emulsify dietary lipids, cholesterol, and fat-soluble vitamin- $\mathrm{s}$ in the small intestine. Synchronously, the gut flora convert parts of the primary bile acids to secondary bile acids such as deoxycholic acid (DCA, derived from CDCA), lithocholic acid (LCA, derived from CA) and a small amount of ursodeoxycholic acid (UDCA, derived from CDCA). About $95 \%$ of bile acids are reabsorbed in the ileum during each cycle of the enterohepatic circulation, and the 5\% that are excreted are replaced by new synthesis in the liver.

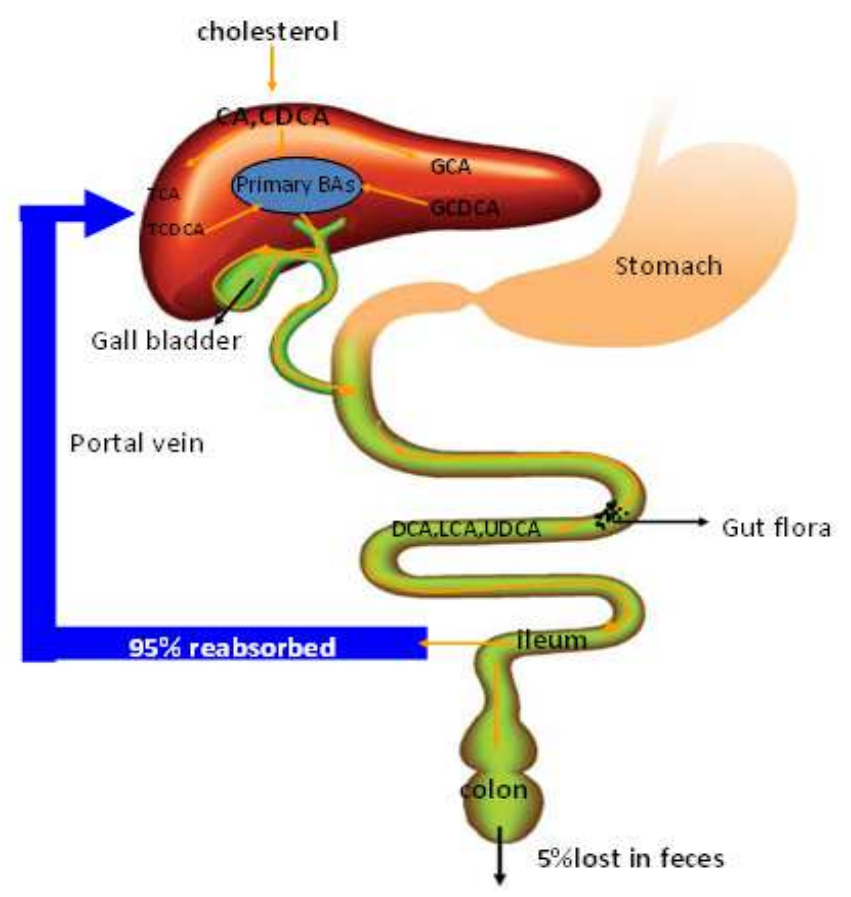

Figure 1: Enterohepatic circulation of bile acids ${ }^{[13-15]}$

\section{Bile Acids and Liver Injury}

Excessive administrations (usually more than 1\%wt/wt) of several hydrophobic BAs (LCA, DCA and CDCA) are reported to be hepatoxic and lead to liver injury on rats, rabbits and primers in the late $1960 \mathrm{~s}^{[16-18]}$. It is generally considered that the toxicity of BAs is correlated with their hydrophobicity. Late studies showed DCA is more toxic than LCA at the same dose, while LCA is more hydrophobic than DCA, suggesting that the hepatotoxicity caused by feeding
BAs does not necessarily depend on the degree of hydrophobicity of the fed BAs ${ }^{[19]}$. Following studies about bile acids explained the possible mechanisms of BAs induced liver injury mainly through apoptosis, necrosis and inflammation ${ }^{[20-22]}$.

\subsection{Inflammation}

Overburdened BAs activated PLC pathways and up-regulated $\mathrm{NF}-\mathrm{\kappa B}$ to produce abundant inflammatory cytokines ${ }^{[23]}$. The mediators such as intercellular adhesion molecular-1 (ICAM-1) and vascular cell adhesion molecule-1 (VCAM-1) were up-regulated by bile acids as well, which is known to be involved in neutrophil trafficking in liver and have been shown to contribute to liver injury ${ }^{[4,24]}$. A recent research shows that TNF- $\alpha$ can enhance the expression of ICAM-1 in the hepatocyte during the inflammatory events ${ }^{[25]}$ Katryn Allen and his colleagues found bile acids act as inflammagens, and may directly activate signaling pathways in hepatocytes by conjugating with a pro-inflammatory mediator: Egr-1 which may directly stimulate the gene expression of ICAM-1 and accumulate neutrophils in the hepatocytes ${ }^{[4]}$.

\section{Bile Acids and Liver Regeneration}

Bile acids are homeotrophic sensors of functional hepatic capacity. It may help quiescent liver cells go through a series of well-orchestrated steps within distinct and recognizable stages of priming, cell cycle progression, proliferation and growth ${ }^{[32]}$. Huang and his colleagues fed wild type mice a relatively mild diet containing $0.2 \%$ cholic acid (CA) for 5 days and their livers' size increased by approximately $30 \%$ without any hepatotoxicity. This work indicates that modest elevated BAs concentrations may drive liver growth and necessary for normal regeneration. Further investigations continuously went on and showed BAs rose in the blood early after $\mathrm{PH}$, and stimulated both hepatocyte proliferation and protection, in part through their binding to the nuclear farnesoid X receptor (FXR) ${ }^{[9]}$.A present study shows that UDCA, a classical specific medicine for cholestatic liver diseases, also benefits in liver regeneration through regulating $\mathrm{G}$ protein-coupled receptor for bile acids (TGR5) and MAPKs pathway ${ }^{[33,34]}$.

\subsection{FXR Pathways}

FXR is an endogenous bile acid receptor and important sensor of liver function [35]. Continuous studies has demonstrated that FXR activation results in the inhibition of CYP7Al, which rapidly down-regulated hepatic bile FXR activation, specifically upregulated ERK pathways and protected liver cells from apoptosis induced by serum deprivation in vitro and fasting in vivo ${ }^{[36]}$. At the same time, FXR promoted liver regeneration for proper liver repair by inducing activation of STAT3, which is a key factor in cell survival ${ }^{[37]}$. Besides, FXR up-regulated the target gene forkhead box M1B (Foxm1b) which was directly involved in 
cell cycle regulation and also in process of the growth hormone (growth hormone, GH) mediating cell proliferation [38]

\subsection{TGR5 Pathway}

BAs activate mitogen-activated protein kinase pathways, and are ligands for the G-protein-coupled receptor (TGR5) ${ }^{[39]}$. TGR5 locates in Kupffer cells induce cell intracellular cyclic adenosine monophosphate (cAMP) synthesis. Activation of TGR5-cAMP pathway benefits liver regeneration through stimulating downstream PKA pathways and inhibiting the expression of inflammatory cytokines stemmed from Kupffer cells ${ }^{[40]}$. TGR5 is also a negative modulator of nuclear factor kappa $(\mathrm{NF}-\kappa \mathrm{B})$ which mediates inflammation ${ }^{[41]}$. Mitsuhiro and his team found TCA may induce enzyme type 2 iodothyronine deiodinase (D2), which converts inactive thyroxine (T4) derived from the fetal bovine serum into active 3,5,3'-tri-iodothyronine (T3) and produces energy, which might supply for liver regeneration [42]

\subsection{MAPKs Pathway}

MAPKs are a class of serine/threonine protein kinases that regulate cell proliferations, apoptosis and other reactions. MAPKs mainly include ERK, c-Jun N-terminal kinase (JNK) and p38MAPK pathways, among which JNK and p38MAKP are called stress-activated protein kinases [43]. Bile acids up-regulated Epidermal Growth Factor Receptor (EGFR) on the membrane and the downstream MAPKs pathways were initiated at once ${ }^{[23]}$. Studies show that JNK and p38MAPK pathways antagonistically control cellular senescence and proliferation ${ }^{[4]}$. Genetic inactivation of the JNK pathway results in impaired proliferation of liver regeneration, which is reversed by inhibition of the p38MAPK. Low concentration of BAs may activate JNK and ERK, but high concentration of BAs may trigger p38MAPK, suggesting that different concentrations of BAs may effect on liver regeneration differently by regulating MAPK pathways.

\section{Application in Drug Development}

Bile acids have been gradually utilized in clinical treatment since UDCA was found effective in dissolving gallbladder stones in 1970s. Many investigators devoted in designing new drugs based on the structure of UDCA. Till now, there are three effective compounds which can be used in cholestatic liver diseases (UDCA, TUDCA and 6- ECDCA).However, UDCA is still the only drug approved by the U.S. Food and Drug Administration (FDA).

Ursodeoxycholic acid (UDCA), a secondary bile acid, is converted from partial CDCA in human colon. It only differs

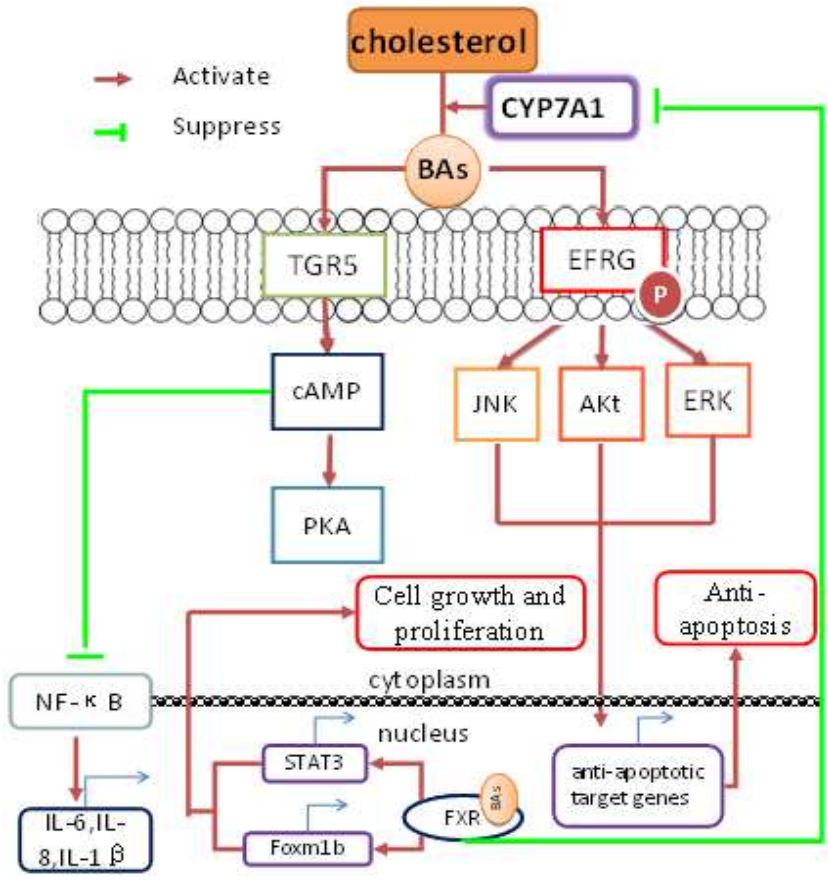

Figure 2: The pathways of liver regeneration ${ }^{[26-31,40-41]}$

from CDCA in the configuration of the hydroxyl group at $\mathrm{C}-7$ ( $\beta$ in UDCA and $\alpha$ in CDCA $)^{[45]}$. However the little difference enhances the hydrophilicity of UDCA and may entitle it with the ability to ameliorate a spectrum of disease rather than cause hepatotoxicity. Early studies show that gallstone dissolution happens in the patients with gallbladder stones treated with UDCA ${ }^{[46]}$. From then on, UDCA has been widely used as therapy for gallstone dissolution, primary biliary cirrhosis (PBC) and other cholestatic diseases ${ }^{[47]}$. As a protective factor, UDCA demonstrated contrary effects to other toxic bile acids, which arouse continuous investigations all over the world for decades. Subsequent researches show that UDCA appears to exert its beneficial effects by stimulating bile

flow, increasing hepatocellular vesicular exocytosis, and reducing the retention of potentially toxic bile acids in hepatocytes ${ }^{[10]}$. Its taurine conjugate tauroursodeoxycholate (TUDCA) has been effectively used for the treatment of cholestatic liver diseases as well ${ }^{[11]}$. Recent studies also show UDCA may play a versatile role in non-cholestatic disease such as colon cancer, or even neurodegenerative diseases through its anti-proliferative and anti-inflammation function [48-50].

But are UDCA and its taurine salts the maverick bile acids which are endowed protective effects? Or the other bile acids have potentially protective function likewise. CDCA, the isomer of UDCA was the first try. Stefano Fiorucci reported that modification of CDCA by addition of an ethyl group in its position 6 results in a semisynthetic bile acid, the 6-ECDCA, has potent FXR agonist activity and has been investigated in preclinical models of cholestasis, liver fibrosis and diet-induced atherosclerosis ${ }^{[12]}$. 
Table 1. bile acids-mediated liver injury and liver regeneration through different pathways.

\begin{tabular}{llll}
\hline Pathway & Function & Main Target & Reference \\
\hline PLC & Induce inflammaion & DAG, IP3, PKC, NF- $\mathrm{KB}$ & {$[23]$} \\
TGR5 & Anti-inflammation & PKA,CREB & {$[39-41]$} \\
MAPK & Induce inflammation (in high concertration) & p38MAPK & {$[23],[40,41]$} \\
FXR & Anti-inflammation (in low concertration) & JNK,AKT, ERK & {$[26-31]$} \\
Egr-1 & Induce proliferation & STAT3,Foxm1b,CYP7A1 & {$[4],[24,25]$} \\
MPT & Induce proliferation & ICAM-1, VCAM-1 & {$[26-31]$} \\
\hline
\end{tabular}

\section{Summary and Perspective}

In this review, we have discussed the toxic and potential protective influences of BAs within the liver through different pathways as is shown in Table 1. Substantial accumulation of BAs will induce severe inflammation, apoptosis and necrosis in hepatocyte, which results in further liver injury. In contrast, not only UDCA, TUDCA and CDCA derivatives have protective effects on liver but also a mild increase of BAs administration may raise liver regeneration through several pathways. Thus, we may assume that BAs play a diverse and dose-dependent role in both liver injury and liver regeneration. The early mechanism of liver injury may be explained that the slight disorders of BAs trigger the beginning of celluar inflammation and apoptosis, while BAs initiate its negative feedback and down-regulation of autosynthesis, which may signal the nuclear receptors and protein kinase to start liver repair. The balance between injury and repair is not broken until the liver regeneration is interpreted due to the overwhelming toxic bile acid accumulation. However, all these hypotheses need further evidence.

\section{Acknowledgements}

This work was supported by National Nature Science Foundation of China (grant number 81222053), Shanghai Nature Science Foundation (grant number 12ZR145300), "Rising-Star Scholar" Project of Shanghai Municipal Science and Technology Commission (grant number 12QH1402200).

\section{References}

[1] Houten, S.M., M. Watanabe, and J. Auwerx, Endocrine functions of bile acids. EMBO J, 2006. 25(7): p. 1419-25.

[2] Li, T. and J.Y. Chiang, Bile Acid signaling in liver metabolism and diseases. J Lipids, 2012. 2012: p. 754067.

[3] Chiang, J.Y., Bile acid metabolism and signaling. Compr Physiol, 2013. 3(3): p. 1191-212.

[4] Allen, K., H. Jaeschke, and B.L. Copple, Bile acids induce inflammatory genes in hepatocytes: a novel mechanism of inflammation during obstructive cholestasis. Am J Pathol, 2011 178(1): p. 175-86.

[5] Geier, A. and C. Trautwein, Bile acids are "homeotrophic" sensors of the functional hepatic capacity and regulate adaptive growth during liver regeneration. Hepatology, 2007. 45(1): p. 251-3.

[6] Monte, M.J., et al., Changes in the pool of bile acids in hepatocyte nuclei during rat liver regeneration. J Hepatol, 2002. 36(4): p. 534-42.

[7] Bhushan, B., et al., Role of Bile Acids in Liver Injury and Regeneration following Acetaminophen Overdose. Am J Pathol, 2013. 183(5): p. 1518-26.

[8] Fausto, N., J.S. Campbell, and K.J. Riehle, Liver regeneration. Hepatology, 2006. 43(2 Suppl 1): p. S45-53.

[9] Huang, W., et al., Nuclear receptor-dependent bile acid signaling is required for normal liver regeneration. Science, 2006. 312(5771): p. 233-6.

[10] Ishizaki, K., T. Imada, and M. Tsurufuji, Hepatoprotective bile acid 'ursodeoxycholic acid (UDCA)' Property and difference as bile acids. Hepatol Res, 2005. 33(2): p. 174-7.

[11] Baumgartner, U., et al., Different protective effects of tauroursodeoxycholate, ursodeoxycholate, and 23-methyl-ursodeoxycholate against taurolithocholate-induced cholestasis. Dig Dis Sci, 1996. 41(2): p. 250-5.

[12] Fiorucci, S., et al., Protective effects of 6-ethyl chenodeoxycholic acid, a farnesoid $\mathrm{X}$ receptor ligand, in estrogen-induced cholestasis. J Pharmacol Exp Ther, 2005. 313(2): p. 604-12.

[13] Russell, D.W., The enzymes, regulation, and genetics of bile acid synthesis. Annu Rev Biochem, 2003. 72: p. 137-74.

[14] Zhang, Y., et al., Effect of bile duct ligation on bile acid composition in mouse serum and liver. Liver Int, 2012. 32(1): p. 58-69.

[15] Rembacz, K., et al., Unconjugated Bile Salts Shuttle through Hepatocyte Peroxisomes for Glycine or Taurine Conjugation. Hepatology, 2008. 48(4): p. 650a-651a.

[16] Hunt, R.D., G.A. Leveille, and H.E. Sauberlich, Dietary Bile Acids and Lipid Metabolism. Iii. Effects of Lithocholic Acid in Mammalian Species. Proc Soc Exp Biol Med, 1964. 115: p. 277-80.

[17] Palmer, R.H., Gallstones Produced Experimentally by Lithocholic Acid in Rats. Science, 1965. 148(3675): p. 1339-40.

[18] Carey, J.B., Jr., et al., The metabolism of bile acids with special reference to liver injury. Medicine (Baltimore), 1966. 45(6): $\mathrm{p}$. 461-70.

[19] Delzenne, N.M., et al., Comparative hepatotoxicity of cholic acid, deoxycholic acid and lithocholic acid in the rat: in vivo and in vitro studies. Toxicol Lett, 1992. 61(2-3): p. 291-304. 
[20] Fiorucci, S., et al., Counter-regulatory role of bile acid activated receptors in immunity and inflammation. Curr Mol Med, 2010. 10(6): p. 579-95.

[21] Schoemaker, M.H., et al., Resistance of rat hepatocytes against bile acid-induced apoptosis in cholestatic liver injury is due to nuclear factor-kappa B activation. J Hepatol, 2003. 39(2): p. 153-61.

[22] Costa, A.M., et al., Role of apoptosis in the remodeling of cholestatic liver injury following release of the mechanical stress. Virchows Arch, 2003. 442(4): p. 372-80.

[23] Lee, H.Y., et al., Bile acid regulates MUC2 transcription in colon cancer cells via positive EGFR/PKC/Ras/ERK/CREB, $\mathrm{PI} 3 \mathrm{~K} / \mathrm{Akt} / \mathrm{IkappaB} / \mathrm{NF}-\mathrm{kappaB}$ and p38/MSK1/CREB pathways and negative JNK/c-Jun/AP-1 pathway. Int J Oncol, 2010. 36(4): p. 941-53.

[24] Gujral, J.S., et al., Functional importance of ICAM-1 in the mechanism of neutrophil-induced liver injury in bile duct-ligated mice. Am J Physiol Gastrointest Liver Physiol, 2004. 286(3): p. G499-507.

[25] Lalor, P.F., et al., Vascular adhesion protein-1 mediates adhesion and transmigration of lymphocytes on human hepatic endothelial cells. J Immunol, 2002. 169(2): p. 983-92.

[26] Palmeira, C.M. and A.P. Rolo, Mitochondrially-mediated toxicity of bile acids. Toxicology, 2004. 203(1-3): p. 1-15.

[27] Chipuk, J.E., et al., The BCL-2 family reunion. Mol Cell, 2010. 37(3): p. 299-310.

[28] Ow, Y.P., et al., Cytochrome c: functions beyond respiration. Nat Rev Mol Cell Biol, 2008. 9(7): p. 532-42.

[29] Fang, Y., et al., Bile acids induce mitochondrial ROS, which promote activation of receptor tyrosine kinases and signaling pathways in rat hepatocytes. Hepatology, 2004. 40(4): p. 961-71.

[30] Booth, D.M., et al., Reactive oxygen species induced by bile acid induce apoptosis and protect against necrosis in pancreatic acinar cells. Gastroenterology, 2011. 140(7): p. 2116-25.

[31] Marin, J.J., et al., Mitochondrial genome depletion in human liver cells abolishes bile acid-induced apoptosis: Role of the Akt/mTOR survival pathway and Bcl-2 family proteins. Free Radic Biol Med, 2013. 61C: p. 218-228.

[32] Khan, A.Z. and S.S. Mudan, Liver regeneration: mechanisms, mysteries and more. ANZ J Surg, 2007. 77(1-2): p. 9-14.

[33] Castro, R.E., et al., Identification of microRNAs during rat liver regeneration after partial hepatectomy and modulation by ursodeoxycholic acid. Am J Physiol Gastrointest Liver Physiol, 2010. 299(4): p. G887-97.

[34] Guicciardi, M.E. and G.J. Gores, Ursodeoxycholic acid cytoprotection: dancing with death receptors and survival pathways. Hepatology, 2002. 35(4): p. 971-3.

[35] Gadaleta, R.M., et al., Bile acids and their nuclear receptor FXR: Relevance for hepatobiliary and gastrointestinal disease. Biochim Biophys Acta, 2010. 1801(7): p. 683-92.
[36] Wang, Y.D., et al., Farnesoid X receptor protects liver cells from apoptosis induced by serum deprivation in vitro and fasting in vivo. Mol Endocrinol, 2008. 22(7): p. 1622-32.

[37] Meng, Z., et al., FXR regulates liver repair after CCl4-induced toxic injury. Mol Endocrinol, 2010. 24(5): p. 886-97.

[38] Chen, W.D., et al., Farnesoid X receptor alleviates age-related proliferation defects in regenerating mouse livers by activating forkhead box $\mathrm{m} 1 \mathrm{~b}$ transcription. Hepatology, 2010. 51(3): p. 953-62.

[39] Pean, N., et al., The receptor TGR5 protects the liver from bile acid overload during liver regeneration in mice. Hepatology, 2013. 58(4): p. 1451-60.

[40] Keitel, V., et al., Expression and function of the bile acid receptor TGR5 in Kupffer cells. Biochem Biophys Res Commun, 2008. 372(1): p. 78-84.

[41] Wang, Y.D., et al., The G-protein-coupled bile acid receptor, Gpbar1 (TGR5), negatively regulates hepatic inflammatory response through antagonizing nuclear factor kappa light-chain enhancer of activated B cells (NF-kappaB) in mice. Hepatology, 2011. 54(4): p. 1421-32.

[42] Watanabe, M., et al., Bile acids induce energy expenditure by promoting intracellular thyroid hormone activation. Nature, 2006. 439(7075): p. 484-9.

[43] Gupta, S., et al., Down-regulation of cholesterol 7alpha-hydroxylase (CYP7A1) gene expression by bile acids in primary rat hepatocytes is mediated by the c-Jun N-terminal kinase pathway. J Biol Chem, 2001. 276(19): p. 15816-22.

[44] Qiao, L., et al., Bile acid regulation of C/EBPbeta, CREB, and c-Jun function, via the extracellular signal-regulated kinase and c-Jun NH2-terminal kinase pathways, modulates the apoptotic response of hepatocytes. Mol Cell Biol, 2003. 23(9): p. 3052-66.

[45] Amaral, J.D., et al., Bile acids: regulation of apoptosis by ursodeoxycholic acid. J Lipid Res, 2009. 50(9): p. 1721-34.

[46] Maton, P.N., G.M. Murphy, and R.H. Dowling, Ursodeoxycholic acid treatment of gallstones. Dose-response study and possible mechanism of action. Lancet, 1977. 2(8052-8053): p. 1297-301.

[47] Soderdahl, G., et al., Ursodeoxycholic acid increased bile flow and affects bile composition in the early postoperative phase following liver transplantation. Transpl Int, 1998. 11 Suppl 1: p. S231-8.

[48] Roma, M.G., et al., Ursodeoxycholic acid in cholestasis: linking action mechanisms to therapeutic applications. Clin Sci (Lond), 2011. 121(12): p. 523-44.

[49] Ros, E., et al., Occult microlithiasis in 'idiopathic' acute pancreatitis: prevention of relapses by cholecystectomy or ursodeoxycholic acid therapy. Gastroenterology, 1991. 101(6): p. 1701-9.

[50] Chun, H.S. and W.C. Low, Ursodeoxycholic acid suppresses mitochondria-dependent programmed cell death induced by sodium nitroprusside in SH-SY5Y cells. Toxicology, 2012. 292(2-3): p. 105-12. 